\title{
EVALUACIÓN DE TRES MÉTODOS DE INJERTACIÓN in vitro EN LA ESPECIE Erythrina edulis (Fabaceae)
}

\section{EVALUATION OF THREE METHODS OF in vitro GRAFTING IN Erythrina edulis SPECIES (Fabaceae)}

\author{
Adriana Paola BONILLA SÁNCHEZ*, Neftalí MESA LÓPEZ \\ Grupo de Investigación en Genética y Biotecnología Vegetal y Microbiana de la Universidad \\ del Tolima (Gebiut). Universidad del Tolima Barrio Santa Helena, Ibagué, Colombia. \\ *Autor corresponsal.E-mail: apbonillas@ut.edu.co
}

Historia del artículo

Recibido: Noviembre 11, 2017

Evaluado: Febrero 14, 2018

Aceptado: Febrero 21, 2018

Disponible: Marzo 16, 2018

\section{Resumen}

Erythrina edulis Triana ex Micheli se considera una especie promisoria, gracias a los valores proteicos de sus hojas y semillas. El estudio se encaminó a la reducción del periodo vegetativo y el aumento en la producción mediante técnicas de injertación. El tratamiento para la desinfección de semillas y la germinación in vitro de embriones consistió en la inmersión de las semillas en alcohol (70\%) durante 15 minutos, seguida de Tween ${ }^{\circledR}(80 \%)$ por 15 minutos, Benlate $(1 \mathrm{~g} / \mathrm{L})$ durante 20 minutos, y HgCl $(0.2 \%)$ por 20 minutos. Como portainjertos se tomaron epicótilos mayores a $3 \mathrm{~cm}$ y como injertos yemas axilares, provenientes de embriones germinados in vitro en medio $\mathrm{MS} 1 / 2+\mathrm{ANA}(2 \mathrm{mg} / \mathrm{L})$. Los injertos se realizaron a través de los métodos aproximación lateral, púa y T invertida, sin modificar el medio. Se realizó una comparación de proporciones al evaluar la desinfección y el prendimiento de los injertos (éxito), y para la variable crecimiento el promedio de tamaño $(\mathrm{cm})$. Los injertos se afectaron por contaminación fúngica y microbiana. El método T invertida presentó altos niveles de oxidación. Se eligió el método aproximación lateral por obtener el mayor porcentaje (29\%) de prendimiento. Para los métodos aproximación lateral y púa se observó un crecimiento promedio de $2.58 \mathrm{~cm}$.

Palabras clave: chachafruto; desinfección; medio de cultivo in vitro; injertación

\section{Abstract |}

Erythrina edulis Triana ex Micheli, is considered a promising species, thanks to the protein values of its leaves and seeds. The study was aimed at reducing the growing season and increased production by grafting techniques. Treatment for seed disinfection and in vitro embryo germination consisted of immersing the seeds in alcohol $70 \%$ for $15^{\prime}$, followed by Tween ${ }^{\circledR} 80 \% 15^{\prime}$, Benlate (1 g/L) for $20^{\prime}$ and $\mathrm{HgCl} 2$ (0.2\%) for $20^{\prime}$. As rootstocks higher epicotyls to $3 \mathrm{~cm}$ were taken as grafts axillary buds, embryos from in vitro germinated amid Ms $1 / 2+$ ANA ( $\left.2 \mathrm{mg} / \mathrm{L}\right)$. The grafts are made through methods (lateral approach, Spikeand and inverted T) without modifying the middle. A comparison of rates was performed, evaluating disinfection and engraftment of linkage (success) to the variable growth and average size (cm). The grafts are affected by fungal and microbial contamination. The inverted T method showed high levels of oxidation. The lateral approach method was chosen to obtain the highest percentage (29\%) of linkage. For methods (Lateral approach and Spike) an average growth of $2.58 \mathrm{~cm}$ was observed.

Keywords: chachafruto; disinfection; in vitro culture medium; grafting

\section{INTRODUCCIÓN}

Erythrina edulis Triana ex Micheli, frijol de árbol o chachafruto, es una leguminosa arbórea originaria de América que crece entre los 1.300 y 2.600 m.s.n.m; en Colombia, se ha observado en la zona cafetera en alturas de hasta $2.200 \mathrm{y}$ 2.500 m.s.n.m. Tradicionalmente, el chachafruto se ha utilizado como especie de interés alimenticio por el destacado valor nutricional y el tamaño de sus semillas, las cuales contienen un 23\% de proteína (Gómez, 2012). 
La biotecnología agrícola se considera una herramienta que permite mejorar la calidad, la seguridad y la sanidad de los productos agrícolas. Además, propicia la búsqueda de una agricultura con un uso racional de agroquímicos y ayuda, de manera sostenible, en la creciente demanda de alimentos (Pasqual et al., 2014). El establecimiento y la estandarización de protocolos in vitro en especies alimenticias son básicos para la implementación de procesos de micropropagación masiva, así como en el propósito de facilitar las técnicas que se emplean en el campo de la biotecnología vegetal (Pasqual et al., 2014). En muchos países, el cultivo in vitro se utiliza con el fin de apoyar programas de mejoramiento genético; al mismo tiempo, la biotecnología genera hoy un extraordinario optimismo con respecto a la capacidad mundial de alimentar a futuras poblaciones, ya que entre los mayores logros de esta ciencia se encuentran obtener plantas libres de enfermedades y la mejora en la adaptación ambiental, entre otras ventajas (CIAT, 2014). Múltiples técnicas se han propuesto en el cultivo in vitro con el objetivo de buscar la solución de problemas presentados en la propagación y el cultivo de plantas. Una de ellas es la injertación in vitro, la cual busca alternativas en la propagación tradicional con miras a reducir el periodo vegetativo, y mejoras en el rendimiento. Aunque es una técnica dispendiosa que exige una manipulación excesiva del explante, ha mostrado buenos resultados en el saneamiento del material propagado y ha contribuido a los estudios básicos de compatibilidad de injertos y a futuros programas de mejoramiento (Navarro et al., 1983).

El objetivo de esta investigación se encaminó a la reducción del periodo vegetativo, el cual, en condiciones de campo, puede durar tres años para la fructificación y el aumento en la producción a través de la técnica de injertación in vitro, con la finalidad de buscar nuevas tecnologías que permitan a futuro contribuir al aumento de la oferta de alimentos ricos en proteína para consumo animal y humano. Se emplearon los tres métodos más representativos de la técnica de injertación y se tuvo en cuenta la planta, el tipo de explante y los objetivos planteados en el estudio.

\section{MATERIALES Y MÉTODOS}

\section{Selección de semillas}

La recolección de semillas se realizó en dos puntos de muestreo: área poblada Pico de Oro, y el corregimiento de Villa Restrepo, ubicados en el Cañón del Combeima, a una altura aproximada de 1.600 m.s.n.m. De los árboles ornamentales seleccionados se tomaron las vainas con buenas características fitosanitarias, como, por ejemplo, ausencia de insectos, sin afectación externa por hongos o bacterias, y sin daños mecánicos.

\section{Tratamientos de desinfección para semillas}

A las legumbres se les realizó un lavado con agua corriente a fin de retirar cualquier partícula contaminante y una limpieza superficial con hipoclorito de sodio al 5.25\%. Luego de la desinfección de las vainas se tomaron las semillas, las cuales se colocaron en etanol al $70 \%$ durante cinco minutos, seguido de un lavado con Tween ${ }^{\circledR}(80 \%)$ por 15 minutos, más una inmersión en Benlate ( $1 \mathrm{~g} / \mathrm{L}$ ) por 20 minutos; posteriormente, se evaluó la acción del $\mathrm{HgCl}_{2}$ en diferentes tiempos de exposición y concentración. Luego de ensayos con 14 tratamientos diferentes, se seleccionaron los cuatro que presentaban mayor posibilidad de éxito, tal como se muestra en la (Tabla 1).

\section{Aislamiento de embriones}

De las semillas desinfectadas se aislaron los embriones intactos para la obtención de vitroplantas, los cuales se inocularon en los medios de cultivo (MS y MS con nitratos a la mitad, ambos suplementados con ácido naftalenacético (ANA) $2 \mathrm{mg} / \mathrm{L}$ ), suplementados con vitaminas y sacarosa al 3\%, pH ajustado a 5.8, una metodología adaptada de Acosta y Zamora (2004). Los embriones se incubaron en oscuridad por un periodo de diez días y, posteriormente, con fotoperiodo de 12 horas luz (lámparas fluorescentes de 1000 lux), a una temperatura de $23-25^{\circ} \mathrm{C}$.

Tabla 1. Tratamientos de desinfección empleados en semilla de E. edulis.

\begin{tabular}{cccccc}
\hline \multirow{2}{*}{$\begin{array}{c}\text { TRATAMIEN- } \\
\text { TOS }\end{array}$} & $\begin{array}{c}\text { ETANOL } \\
\text { 70\% }\end{array}$ & TWEEN & BENLATE & \multicolumn{2}{c}{$\mathrm{HGCL}_{2}$} \\
\cline { 5 - 6 } & & & $\mathbf{1 G} / \mathrm{L}$ & $\mathbf{0 . 2 \%}$ & $\mathbf{0 . 3 \%}$ \\
\hline 1 & & & & $10 \mathrm{~min}$ & - \\
3 & $5 \mathrm{~min}$ & $15 \mathrm{~min}$ & $20 \mathrm{~min}$ & $20 \mathrm{~min}$ & \\
$4^{*}$ & & & & - & $20 \mathrm{~min}$ \\
& & & & $20 \mathrm{~min}$ &
\end{tabular}

*Medio suplementado con 2 ml/L de PPM (Plant Preservative Mixture)

\section{Tratamiento para la multiplicación de portainjertos}

Una vez establecidos los embriones de E. edulis y alcanzado un número considerable de vitroplantas, se realizó una etapa de multiplicación al subdividir la plántula según los brotes y yemas formados, y así tomar segmentos nodales con una yema axilar, los cuales se sembraron en medio nutritivo MS con la concentración de nitratos a la mitad de la formulación básica con una combinación de benciloaminopurina (BAP) y ANA a (1:1 mg/L). Los tallos que sirvieron como portainjerto presentaban un diámetro de 0.2 a $0.3 \mathrm{~mm}$.

\section{Procedimiento de injertación}

Los procesos previos a la realización del injerto, como, por ejemplo, los métodos de incisión, se ajustaron según la metodología propuesta por Juárez y Navarro (2005).

\section{Preparación del injerto}

Yemas axilares de aproximadamente $2 \mathrm{~mm}$ de longitud fueron utilizadas como injerto. Estas se tomaron a partir de plantas madre de seis meses de edad, producidas in vitro a partir del aislamiento de embriones por organogénesis directa en los medios de cultivo antes especificados.

\section{Injertación}

En el procedimiento se usaron vitroplantas de tres meses de edad, las cuales se podaron en su parte apical, de modo que se dejó una porción del epicótilo de 3-4 cm, se eliminaron 
sus yemas axilares y una porción del ápice de la raíz, y se dejó un trozo de $2-3 \mathrm{~cm}$.

\section{Métodos de incisión}

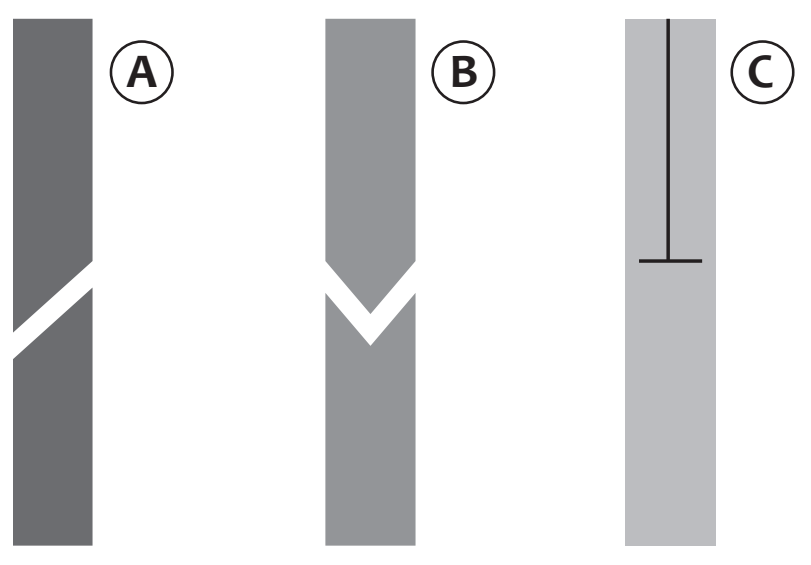

Figura 1. Tipos de incisión:

A) Aproximación lateral. B) Púa y C) T invertida.

\section{Aproximación lateral}

A las yemas vegetativas asépticas "injerto" se les realizó un corte en forma de bisel en la parte basal, a fin de que entrara en contacto con la zona vascular de la plántula podada "portainjerto", la cual también presentaba una incisión en bisel (Fig. 1A).

\section{Púa}

Se tomaron brotes vegetativos de 2 a $3 \mathrm{~cm}$ de longitud, con presencia de yemas; se les realizó un corte en forma de "v" invertida en la parte terminal. A los portainjertos se les eliminaron las hojas, y luego en la porción basal de estos se realizaron dos cortes para formar una "v" o cuña y, de esta manera, acoplar las yemas dentro de la hendidura del portainjerto (Fig. 1B). En los dos métodos descritos el contacto entre el injerto y el portainjerto se mantuvo con la utilización de anillos de silicona estériles.

\section{T invertida}

Se realizó un corte en forma de T invertida (Fig. 1C) en la parte superior del patrón, en cuya incisión se colocó la yema vegetativa o injerto, y se aseguró así que los laterales del corte abrazasen por completo a la yema.

Los injertos se cultivaron en medio MS con concentración de fuentes de nitratos a la mitad de la concentración propuesta en la formulación básica, suplementado con $2 \mathrm{mg} / \mathrm{L}$ de ANA, con el fin de observar el desarrollo de las yemas injertadas sobre el portainjerto.

\section{Análisis estadístico}

En este estudio se consideraron las siguientes variables de tipo categórico: A. Contaminación con las categorías: 1. Presencia de contaminación (hongos y bacterias); y 2. Ausencia de contaminación. B. Oxidación con las categorías: 1. Presen- cia de oxidación (producción fenólica); y 2. Ausencia de oxidación. C. Prendimiento con las categorías: 1. Formación de callo; y 2 . Ausencia de callo.

Las comparaciones de los tratamientos se realizaron mediante diseño estadístico de comparación de proporciones. Este diseño se escogió dada la naturaleza de las variables consideradas, la cuales no permiten otro tipo de comparaciones (por ejemplo, mediante análisis de varianza). Se analizaron los factores contaminación, oxidación y prendimiento. La estimación de las proporciones, al tener en cuenta que los tamaños de muestra son pequeños, se realizó con el método exacto de Blyth y Hutchinson (1960), el cual produce un intervalo de la forma $(\pi i, \pi \mathrm{s})$ donde:

$$
\begin{gathered}
\pi i=\frac{x}{x+(n-x+1) F i} \operatorname{con} F i=F 2(n-x+2), 2 x, \alpha / 2 \\
\pi s=\frac{(x+1) F s}{n-x+(x+1) F s} \operatorname{con} F s=F 2(x+1), 2(n-x), \alpha / 2
\end{gathered}
$$

En la expresión anterior $n$ es el tamaño de muestra usado y $x$ el número de éxitos presentados en la muestra. Fi, Fs son valores correspondientes a los cuantiles que determinan una cola de probabilidad de $\frac{\alpha}{2}$ con $\alpha=0.05$ bajo la distribución $\mathrm{F}$, con los grados de libertad expresados en las fórmulas anteriores (EC. 1). Se utilizó un mínimo de 15 explantes por tratamiento; sin embargo, en algunos ensayos se optó por aumentar el número de observaciones, ya que por ser un método exacto de construcción de intervalos no dependen del tamaño muestral. Se consideró que existían diferencias entre proporciones si y solo si sus correspondientes intervalos de confianza presentaban "intersección vacía”, y esto se aplicó para las comparaciones de todos los pares de tratamientos. En el cálculo de los intervalos de confianza se hizo uso de la calculadora en línea del sitio web http.statpages.org, y hojas de cálculo de Microsoft Excel.

Las respuestas de tipo cuantitativas tales como número de brotes y magnitud del crecimiento $(\mathrm{cm})$ se evaluaron mediante la estimación de los respectivos promedios, con un nivel de confianza del 95\%; por su parte, la construcción de los respectivos intervalos con el programa estadístico Infostat versión estudiantil.

\section{RESULTADOS Y DISCUSIÓN}

Efecto de los tratamientos de desinfección sobre el establecimiento in vitro del embrión

De acuerdo con las pruebas estadísticas de comparación de proporciones, con un $95 \%$ de confiabilidad se evidenció que los tratamientos más efectivos para lograr la asepsia de los embriones correspondió a los tratamientos T2, T3 y T4, con lo cual se comprueba la efectividad del dicloruro de mercurio para controlar la contaminación bacteriana, como lo afirman Beltrán y Mesa (2014) en yemas in vitro de Aniba perutilis Hemsley en igual concentración y tiempo de exposición. Se considera al T2 ( $\mathrm{HgCl} 2$ 0.2\% por 20 minutos) como el más sobresaliente, con 
una estimación puntual de (0.14) (ó 14\% de contaminación), aun cuando este tratamiento no difiere del T4 y el T3, ya que los correspondientes intervalos de confianza se traslapan (Tabla 2). En esta investigación se descarta el tratamiento ( $\left.\mathrm{T}_{1}\right)$, ya que con una estimación puntual de 0.75 (ó 75\%) mostró ser el menos efectivo en su acción antibacteriana.

Efecto del medio nutritivo sobre el desarrollo de los embriones in vitro

Los dos medios de cultivo propuestos para el establecimiento de los embriones de chachafruto resultaron ser efectivos para obtener plántulas completas, sin embargo, se optó por emplear el medio MS (concentración de nitratos a la mitad) suplementado con ANA ( $2 \mathrm{mg} / \mathrm{L})$, por arrojar una mayor elongación de la parte apical, buen desarrollo en la parte radical, así como un mayor número de entrenudos por vitroplanta y una vigorosidad y coloración intensa.

Tabla 2. Estimación puntual de proporciones de contaminados en los tratamientos de desinfección de E. edulis.

\begin{tabular}{cccc}
\hline TRATAMIENTOS & $\mathbf{N}$ & $\begin{array}{c}\text { ESTIMACIÓN } \\
\text { PUNTUAL } \\
\text { DE LA } \\
\text { PROPORCIÓN }\end{array}$ & $\begin{array}{c}\text { INTERVALO DE } \\
\text { CONFIANZA } \\
\text { DEL 95\% }\end{array}$ \\
\hline T2 & 34 & $5 / 34=0.14$ & $(0.0495-0.3106)$ \\
$T 4$ & 35 & $13 / 35=0.37$ & $(0.2147-0.5508)$ \\
$T 3$ & 15 & $7 / 15=0.46$ & $(0.2127-0.7341)$ \\
$T 1$ & 20 & $15 / 20=0.75$ & $(0.5090-0.9134)$
\end{tabular}

Existen diferencias entre proporciones si y solo si los intervalos de confianza presentan "intersección vacía”.

En relación con este resultado, Abedini et al. (2000) afirman que el uso de medios de cultivo, con las concentraciones completas de nitratos en leguminosas como Erythrina crista-galli L., disminuye de forma sustancial la expresión del material vegetal en condiciones in vitro, debido, entre otras causas, al aumento de la oxidación y al desorden fisiológico que se da como consecuencia de la hiperhidratación. Estos autores también destacan la importancia de adicionar al medio de cultivo fitorreguladores de tipo auxínico, pues participan y aceleran el proceso de germinación. De allí su importancia en ensayos en los que se aísla el embrión de su endospermo, o se aplican técnicas como, por ejemplo, el rescate de embriones.

\section{Multiplicación de los portainjertos}

Se seleccionó el medio nutritivo MS con la concentración de nitratos a la mitad de la formulación básica, con una combinación de BAP y ANA a (1:1 mg/L). Para la multiplicación masiva de portainjertos en este medio se estimó el promedio del número de brotes por cada unidad experimental (Tabla 3), lo cual arrojó un promedio de número de brotes de 2.58 en un periodo de dos meses con un intervalo de confianza del $95 \%$, y valores entre 1.80 y 3.37. Esto ratifica la respuesta positiva del explante "segmento nodal" al medio seleccionado, al estimular la formación de múltiples brotes y favorecer el crecimiento de las ramas axilares y laterales.
Tabla 3. Estimación del promedio de brotes para la construcción del intervalo de confianza.

\begin{tabular}{|c|c|c|c|c|c|c|}
\hline \multicolumn{7}{|c|}{ BILATERAL } \\
\hline \multicolumn{7}{|c|}{ ESTIMACIÓN PARAMÉTRICA } \\
\hline Variable & Parámetro & Estimación & E.E. & $\mathrm{n}$ & LI (95\%)) & LS $(95 \%)$ \\
\hline Brotes $2 \mathrm{~g}$ & Media & 2,58 & 0,36 & 12 & 1,80 & 3,37 \\
\hline
\end{tabular}

Aunque se reporta que la citoquinina tiene un efecto de supresión sobre la acción de las auxinas, se ha encontrado que la utilización de concentraciones precisas de las dos hormonas puede tener un efecto sinérgico en la formación de brotes (Azizi et al., 2015). Asimismo, en especies forestales como el comino $A$. perutilis (Beltrán y Mesa, 2014) se da un desarrollo caulinar por efecto del BAP, pero se presenta inhibición en el proceso de enraizamiento (aun en presencia de ANA en concentración $1 \mathrm{mg} / \mathrm{L}$ ). Esto coincide con lo observado en este estudio, ya que hubo presencia de una masa tipo callosa en la zona de base caulinar.

\section{Injertación in vitro de Erythrina edulis}

La viabilidad de los injertos se vio comprometida por una alta contaminación fúngica posterior a los 12 días de realizar la unión. Aunque en la consolidación del injerto se emplearon explantes asépticos producidos in vitro, esto no fue garantía de éxito en la etapa de su establecimiento, pues los niveles de contaminación fueron del $66 \%, 70 \%$ y $76 \%$ al emplear los métodos de incisión T invertida, púa y aproximación lateral (Tabla 4A). Respecto a la variable oxidación, se encontró diferencia significativa entre los métodos T invertida y púa; fue el primero el que registró un mayor nivel de oxidación con una estimación puntual de 0.80 (ó $80 \%$ ), tal como se presenta en la (Tabla 4B), lo que impidió el prendimiento del injerto y, por tanto, su evaluación como método de injertación.

La alta contaminación y oxidación de los injertos puede explicarse, posiblemente, por la excesiva manipulación de los explantes durante la realización de las incisiones y su posterior unión (Rache et al., 2008; Materán et al., 2008). En este punto es importante resaltar que los portainjertos se produjeron a partir de aislamiento de embriones - lo que disminuye el diámetro del tallo-, por lo que autores como Hussainet et al. (2014) proponen la obtención de portainjertos a partir de germinación de la semilla in vitro.

Tabla 4. Estimación puntual de las proporciones analizando A) la contaminación, y B) oxidación, en los tres métodos de injertación.

\begin{tabular}{cccc}
\hline TRATAMIENTOS A & N & $\begin{array}{c}\text { ESTIMACIÓN } \\
\text { PUNTUAL DE LA } \\
\text { PROPORCIÓN }\end{array}$ & $\begin{array}{c}\text { INTERVALO DE } \\
\text { CONFIANZA } \\
\text { DEL 95\% }\end{array}$ \\
\hline Tinvertida & 15 & $10 / 15=0.66$ & $(0.3838-0.8818)$ \\
Púa & 17 & $13 / 17=0.76$ & $(0.5010-0.9319)$ \\
$\begin{array}{c}\text { Aproximación } \\
\text { lateral }\end{array}$ & 17 & $12 / 17=0.70$ & $(0.4404-0.8969)$ \\
& & $\begin{array}{c}\text { Estimación } \\
\text { puntual de la } \\
\text { proporción }\end{array}$ & $\begin{array}{c}\text { Intervalo de confianza } \\
\text { del 95\% }\end{array}$ \\
& & Continúa en próxima página »
\end{tabular}




\begin{tabular}{cccc} 
Tinvertida & 15 & $12 / 15=0.80$ & $(0.5191-0.9567)$ \\
Púa & 17 & $4 / 17=0.23$ & $(0.0681-0.4990)$ \\
Aproximación lateral & 17 & $5 / 17=0.29$ & $(0.1031-0.5596)$ \\
\hline
\end{tabular}

Existen diferencias entre proporciones si y solo si los intervalos de confianza presentan "intersección vacía”.

\section{Efecto del tipo de incisión en el prendimiento de los injertos}

En los injertos que superaron la contaminación y mostraron un desarrollo de las yemas injertadas se evaluó si el tipo de incisión favorecía el prendimiento del injerto, así como su capacidad de adaptación. La (Tabla 5) refleja que a través de los métodos de injertación de púa y aproximación lateral se logró un prendimiento promedio del 0.23 y 0.29 , respectivamente (ó $23 \%$ y $29 \%$ de prendimiento), a diferencia del método de T invertida, en el que no fue posible observar la presencia de hojas ni el desarrollo de las yemas injertadas por efecto de la oxidación y la contaminación. Sin embargo, el análisis de comparación de proporciones —al evaluar el prendimiento - no reflejó, estadísticamente, diferencias significativas entre los tres métodos.

En el caso puntual de los métodos de aproximación lateral y el de púa se logró observar, en los injertos que prosperaron, la formación de un callo translucido a las tres semanas de realizada la injertación. Según lo referenciado por Juárez (2013), al injertarse dos especies, las partes afectadas, inicialmente desarrollan un callo — producto de la intensa actividad mitótica- que posibilita la unión. Por esto se seleccionó el método que obtuvo mayor prendimiento y facilidad para su implementación, como lo fue el de aproximación lateral.

Tabla 5. Estimaciones puntuales de prendimiento en injertos de $E$. edulis en tres métodos de injertación.

\begin{tabular}{cccc}
\hline PRENDIMIENTO & $\mathbf{N}$ & $\begin{array}{c}\text { ESTIMACIÓN } \\
\text { PUNTUAL } \\
\text { DE LA PROPORCIÓN }\end{array}$ & $\begin{array}{c}\text { INTERVALO DE } \\
\text { CONFIANZA DEL 95\% }\end{array}$ \\
\hline Tinvertida & 15 & $0 / 15=0$ & $(0.0000-0.2180)$ \\
Púa & 17 & $4 / 17=0.23$ & $(0.0681-0.4990)$ \\
$\begin{array}{c}\text { Aproximación } \\
\text { lateral }\end{array}$ & 17 & $5 / 17=0.29$ & $(0.1031-0.5596)$
\end{tabular}

Existen diferencias entre proporciones si y solo si los intervalos de confianza presentan “intersección vacía”.

Respecto al bajo rendimiento presentado en el método T Invertida, Navarro (1979) señala que el método de colocación del ápice en el patrón influye de forma significativa en el grado de prendimiento. De igual manera, este autor reporta que al colocar el ápice dentro de la incisión tipo T Invertida, pero con su superficie basal de corte en contacto con el tejido de la médula, dio un bajo porcentaje de prendimiento. Esto se debe a que el injerto no queda realmente en contacto directo con el tejido vascular, sino con tejido medular.

El medio básico MS (con nitratos a la mitad), suplementado con ANA (2 mg/L), permitió el desarrollo de las plantas in- jertadas, de modo que se logró estimular el crecimiento de las yemas vegetativas del injerto 45 días después de realizada la incisión. Este comportamiento puede estar influenciado por la acción hormonal del ANA. Ayala (2011) considera que su presencia promueve la organogénesis e induce la generación de callo como parte del rejuvenecimiento, o en respuesta a heridas por cortes y manipulación.

Tabla 6. Estimación del promedio crecimiento de yemas en el injerto para la construcción del intervalo de confianza.

\begin{tabular}{lcccccc}
\hline \multicolumn{1}{l}{ BILATERAL } \\
\hline \multicolumn{10}{l}{ ESTIMACIÓN PARAMÉTRICA } \\
\hline Variable & Parámetro & Estimación & E.E. & $\mathrm{n}$ & LI (95\%)) & LS (95\%)) \\
Crecimiento & Media & 1,58 & 0,40 & 6 & 0,56 & 2,61
\end{tabular}

Por otra parte, el análisis estadístico (Tabla 6) permite inferir que, en un lapso de 45 días, el crecimiento promedio de las yemas injertadas fue de $1.58 \mathrm{~cm}$, con un intervalo de confianza del $95 \%$ dado entre 0.89 y 2.22 . Este crecimiento se considera un indicador morfológico que ratifica la unión de los tejidos en el injerto. Criollo (2008) señala que el medio ideal para cualquier injerto es el que permite un desarrollo del portainjerto en su estructura y en su sistema radical, lo cual garantiza la vitalidad de este. En razón a lo anterior, se considera que el medio MS (nitratos a la mitad) + ANA (2 mg/L) cumple con estos criterios.

\section{CONCLUSIONES}

La desinfección más efectiva para controlar la contaminación de los embriones a partir de semillas de Erythrina edulis fue: alcohol (70\%), durante 15 minutos; seguida de Tween ${ }^{\circledR}$ (80\%) por 15 minutos; Benlate $(1 \mathrm{~g} / \mathrm{L})$ durante 20 minutos; y dicloruro de mercurio (0.2\%) por 20 minutos. La reducción de la concentración de nitratos a la mitad en el medio de cultivo MS con la adición de ANA $2.0 \mathrm{mg} / \mathrm{L}$ permitió la obtención de plántulas completas de chachafruto; de igual manera, fue el medio de cultivo seleccionado para el prendimiento del injerto. Se selecciona el método de aproximación lateral para la injertación por ser el de mayor prendimiento y facilidad de ejecución. La injertación in vitro con los métodos de púa y T Invertida deben excluirse hasta tanto no se logre controlar la contaminación y la oxidación.

\section{AGRADECIMIENTOS}

Los autores agradecen al Comité Central de Investigaciones de la Universidad del Tolima por la financiación de este proyecto, así como a los integrantes del Grupo Gebiut y al profesor Jairo Alfonso Clavijo MSC., por su valiosa colaboración y asesoría estadística.

\section{REFERENCIAS}

- Abedini, W. P., Boeri, P., Marinucci, L., Ruscitti, M. y Scelzo. L. (2000). Biotecnologías aplicadas a especies forestales nativas. Investigación agraria. Sistemas y Recursos Forestales, 9(1), 31-35. 
- Acosta, M. y Zamora, M. (2004). Aislamiento de protoplastos del mesofilo de hoja de vitroplantas de chachafruto (Erythrina edulis) (Trabajo de grado). Programa de Biología, Facultad de Ciencias, Universidad del Tolima, Ibagué.

- Ayala, A. N. (2011). Establecimiento de cultivo in vitro de Molle (Schinus molle L.) a partir de yemas axilares tomadas de plantas madre como una herramienta para la propagación de la especie en el distrito metropolitano de Quito (Trabajo de grado). Programa de Ing. en Biotecnología, Departamento de Ciencias de la Vida e Ingeniería en Biotecnología, Escuela Politécnica del Ejército, Sangolquí, Ecuador.

- Azizi, P., Rafii, M. Y., Maziah, M, Abdullah, S. N. A., Hanafi, M. M., Latif, M. ... y Sahebi, M. (2015). Understanding The Shoot Apical Meristem Regulation: A Study of The Phytohormones, Auxin and Cytokinin, in Rice. Mechanisms of Development, 135,1-15. doi: 10.1016/j.mod.2014.11.001.

- Blyth, C. R. y Hutchinson, D. W. (1960). Table of Neyman-Shortest Unbiased Confidence Intervals for the Binomial Parameter. Biometrika, 47(3-4), 381-391.

- Beltrán, D. M. y Mesa, N. (2014). El dicloruro de mercurio como desinfectante en la micropropagación del comino (Aniba perutilis Hemsley). Revista Colombiana de Biotecnología, 16(1), 203-209. doi: https://doi.org/10.15446/rev. colomb.biote.v16n1.44302.

- CIAT (Centro Internacional de Agricultura Tropical). (2014). CIAT Strategy 20142020: Buildingan eco-efficient future. Cali, Colombia: CIAT.

- Criollo, D. L. (2008). Evaluación de dos técnicas para la microinjertación de babaco (Vasconcellea heilbornii cv. pentagona) y chihualcán (Vasconcellea heilbornii cv. chrysopetala) en patrones de papaya (Carica papaya) bajo condiciones de laboratorio, Santa Catalina-Iniap (Trabajo de grado). Programa de Ing. en Biotecnología, Departamento de Ciencias de la Vida e Ingeniería en Biotecnología, Escuela Politécnica del Ejército, Sangolquí, Ecuador.

- Gómez, A. (2012). Caracterización con marcadores moleculares RAM's (Random Amplified Microsatellites) de algunas especies del género Erythrina presentes en Colombia (Tesis de maestría). Facultad de Ciencias Agropecuarias, Universidad Nacional de Colombia, Palmira, Valle del Cauca.

- Hussain, G., Wani, M. S., Mir, M. A., Rather, Z. A. y Bhat, K. M. (2014). Micrografting for fruit crop improvement. African Journal of Biotechnology, 13(25), 2474-2483. doi:10.5897/AJB2013.13602.

- Juárez, J. (2013). Rejuvenecimiento de Cedro Rojo (Cedrela odorata L.), mediante microinjertación e injertación (Tesis de maestría). Posgrado en Ciencias Biológicas, Unidad de Biotecnología, Centro de Investigación Científica de Yucatán, A. C., Mérida, México.

- Juárez, J. y Navarro, L. (2005). Microinjerto de ápices caulinares de cítricos "in vitro". Phytoma, 170, 55-65.

- Materán, M. E., Vega, M. C., Sánchez, M., Sáez, K., Rodríguez, R. y Ríos, D. (2008). Reactivación de material vegetal élite de Pinus radiata D. Don. mediante microinjertación in vitro. Interciencia, 33(1), 66-70.

- Navarro, L. (1979). Microinjerto de ápices caulinares in vitro para la obtención de plantas de agrios libres de virus. BOL. Serv. Plagas, 5, 127-148.

- Navarro, L., Llácer, G., Cambra, M., Arregui, J. M. y Juárez, J. (1983). Shoottip grafting in vitro for elimination of viruses in peach plants (Prunus persica Batsch). Acta Hortic, 130, 185-192. doi: 10.17660/ActaHortic.1983.130.32.

- Pasqual, M., Rodrigues-Soares, J. D. y Almendagna-Rodrigues, F. (2014). Tissue Culture Applications for the Genetic Improvement of Plants. En A. Borém y R. Fritsche-Neto (Eds.), Biotechnology and Plant Breeding: Applications and Approaches for Developing Improved Cultivars (pp. 157-178). Oxford (RU): Elsevier.

- Rache, L., Rojas, E. y Pacheco J. (2008). Revigorización y clonación de yemas adultas de árboles de olivo: establecimiento in vitro de microinjertos. Bioagro, $20(1), 57-65$. 